\title{
Correlation of Salivary Resistin Levels with Obstructive Sleep Apnea Syndrome in Pediatric Subjects
}

\author{
Jae Hyung Hwang, MD', In Hye Kim, MD¹, Hye Sook Lee, MD', \\ Dong Sun Park, MD, PhD², Chan-Soon Park, MD, PhD \\ 'Department of Otolaryngology-Head and Neck Surgery, St. Vincent's Hospital, College of Medicine, The Catholic University of Korea, Suwon, Korea \\ ${ }^{2}$ Department of Otolaryngology-Head and Neck Surgery, CEO Hospital, Suwon, Korea
}

Received: October 16, 2018

Revised: December 19, 2018

Accepted: December 20, 2018

Correspondence

Chan-Soon Park, MD, PhD

Department of Otorhinolaryngology-Head

and Neck Surgery, St. Vincent's Hospital,

College of Medicine,

The Catholic University of Korea,

93 Jungbu-daero, Paldal-gu,

Suwon 16247, Korea

Tel +82-31-881-8968

Fax +82-31-257-3752

E-mail pcs0112@catholic.ac.kr

ORCID

Jae Hyung Hwang

https://orcid.org/0000-0001-7249-0318 In Hye Kim

https://orcid.org/0000-0001-6511-6950

Hye Sook Lee

https://orcid.org/0000-0002-5903-696X

Dong Sun Park

https://orcid.org/0000-0003-3531-7422

Chan-Soon Park

https://orcid.org/0000-0003-3692-3344
Background and Objective Obstructive sleep apnea syndrome (OSAS) is considered to be closely related to systemic inflammation. Resistin levels have been demonstrated to be a measure of systemic inflammation. For children, salivary resistin (SR) sampling is an easy and pain-free method for sample collection and is optimal for multiple sampling. Therefore, we aimed to evaluate correlations among SR levels, objective polysomnography (PSG) parameters, and subjective sleep symptoms.

Methods Fifty-six children who attended our clinic over 1 year were enrolled prospectively; these children underwent clinical evaluation, questionnaire studies, and PSG. SR was measured at 2 points: at night before PSG and in the early morning after PSG.

Results The subjects $(\mathrm{n}=56)$ were divided into the control $[\mathrm{n}=23$, apnea hypopnea index $(\mathrm{AHI})<1]$ and OSAS $(\mathrm{n}=33, \mathrm{AHI} \geq 1)$ groups. SR levels after PSG in the OSAS and control groups were similarly higher than that before PSG. There was no significant difference in SR levels between the two groups and among the control and OSAS subgroups. SR levels in the OSAS subgroups were not related to $\mathrm{AHI}$, tonsil size, AN (adenoid-nasopharyngeal) ratio, questionnaire results, lowest oxygen saturation, and oxygen desaturation index (ODI).

Conclusions SR levels had no significant correlation with AHI, scores of the Korean version of modified pediatric Epworth Sleepiness Scale, the lowest oxygen saturation, and ODI; SR levels exhibited diurnal variations regardless of the presence of OSAS. However, there is no consensus regarding the relationship between resistin levels and OSAS. Further studies should be pursued in the future.

Sleep Med Res 2018;9(2):77-82

Key Words Apneas, Obstructive sleep, Saliva, Resistin.

\section{INTRODUCTION}

Pediatric obstructive sleep apnea syndrome (OSAS) is one of the most frequently diagnosed pediatric sleep disorders [1,2]. OSAS is associated with neurocognitive impairment, behavioral problems, failure to thrive, hypertension, and cardiac dysfunction [3]. OSAS-related lowgrade systemic inflammation and increased oxidative stress are believed to underpin the development of these OSAS-related morbidities [1,4].

Resistin is cysteine-rich secretory protein and was initially described as an adipokine that increases insulin resistance. Resistin is associated with human obesity and has been recently identified as a novel important member of the cytokine family involved in the regulation of inflammation and is reported to have potent regulatory function. Adipocytes, circulating monocytes, and macrophages may be responsible for resistin production in humans [5,6]. Although there have been some controversies regarding the pathophysiological mechanisms underlying OSAS in children, recent studies have showed correlations between fragmented sleep and an 
increase in oxidative stress mediators [6-8]. Intermittent episodes of hypoxia, along with reoxygenation, in OSAS patients might cause cell stress through the activation of a proinflammatory response [9].

The human saliva mirrors the body's health and can be collected noninvasively. Moreover, saliva sampling does not require specialized skills and is suitable for large populationbased screening programs [10]. Thus, it can be said that sleepdisordered breathing (SDB) with high prevalence may be a good candidate for salivary markers. Recently, a few studies have reported that measurements of salivary cortisol and amylase in children might help identify those with high apnea hypopnea index (AHI) and prioritize the management of such children $[11,12]$. Elevated circulating adipokine levels have been reported in adults and children with OSAS; furthermore, adipokines, such as adiponectin, leptin, resistin, and visfatin, can be detected in the saliva of healthy subjects [10]. Therefore, our hypothesis in this study is that salivary levels of adipokines such as resistin might be related to SDB and its severity, and thus, these salivary adipokines could be good diagnostic markers for SDB in children. To the best of our knowledge, to date, no data on saliva resistin (SR) levels in pediatric SDB have been reported. The aim of this study was to measure SR levels in newly diagnosed pediatric SDB patients and to find the correlation of SR levels with SDB and SDB severity.

\section{METHODS}

\section{Study Design and Subjects}

Between July 2013 and June 2015, 56 children (aged 3-13 years) with suspected OSAS visited the department of Otolaryngology-Head and Neck Surgery, St. Vincent's Hospital, Suwon, Republic of Korea and were enrolled in this study prospectively. Exclusion criteria were other causes of airway obstruction such as rhinitis, antrochoanal polyp, nasal polyposis, congenital anomaly with craniofacial abnormalities, neuromuscular dystrophies, and prior operation or diagnostic history of cardiac or airway disease.

In our previous studies [11-13], we described the physical and radiological findings, polysomnography (PSG), and experimental methods for salivary markers for SDB. The study protocol was approved by the Institutional Review Board of the Catholic Medical Center Clinical Research Coordinating Center, and informed consent was obtained from all participants at the time of enrolment. The steps and procedures applied in this study have been described in our previous studies and can be briefly described as follows.

All children underwent a complete physical examination. The size of each tonsil was measured as the sum of each subjective tonsil size scale [11] by one doctor because some subjects might have asymmetric tonsillar sizes. To evaluate adenoid size, we measured the adenoidal-nasopharyngeal (AN) ratio in the lateral neck radiograph or cephalometry according to Fujioka et al. [14] method. Parents of all children completed the Korean version of modified pediatric Epworth Sleepiness Scale (KMPESS). Fully attended-PSG was performed, and all measured parameters were scored initially by a certified technician and then reviewed by a doctor. The collection of the saliva in the enrolled children was performed two times: before PSG and after PSG. The study protocol was reviewed and approved by the Ethics Committee for Clinical Studies of St. Vincent's Hospital, the Catholic University of Korea (VC14TISI0016).

\section{Measurement of Salivary Resistin}

Saliva samples were collected in special tubes around 10:00 pm prior to PSG and at 7:00 the next morning after PSG (within 30 minutes of waking up). Whole unstimulated saliva was collected by tilting the head forward, allowing the saliva to pool on the floor of the mouth, and then passing the saliva through a short straw into a polypropylene vial. Then, the samples were refrigerated within 30 minutes and frozen at or below $-20^{\circ} \mathrm{C}$ within 4 hours after collection. On the day of assay, they were thawed completely, vortexed, and centrifuged at $1500 \times \mathrm{g}(3000$ $\mathrm{rpm}$ ) for 15 minutes. Samples were kept at room temperature before adding to the assay plate. All samples were assayed in duplicate for SR using a highly sensitive enzyme immunoassay (EK028-36, Phoenix Pharmaceuticals Inc., Burlingame, CA, USA).

\section{Statistics}

All statistical tests were conducted using the Statistical Package for the Social Sciences (SPSS Inc., Chicago, IL, USA). The Shapiro-Wilk test was performed to assess the normality of the data before further analysis. Quantitative variables in the case $(\mathrm{AHI} \geq 1)$ and control $(\mathrm{AHI}<1)$ groups were compared using the t-test or Wilcoxon rank-sum test. Pearson's or Spearman's correlations were applied to determine the relationship of the SR measurements with parameters of PSG and subjective symptoms (from the questionnaire). A p value $<0.05$ was considered to indicate statistical significance.

\section{RESULTS}

The subjects $(\mathrm{n}=56)$ were divided into control $(\mathrm{n}=23$, AHI < $1)$ and OSAS ( $n=33, \mathrm{AHI} \geq 1)$ groups. The demographic data and PSG findings are presented in Table 1 . The OSAS group $(\mathrm{n}=$ 33) was subdivided into three groups: mild ( $\mathrm{n}=15,45.4 \% ; 1 \leq$ AHI $<5)$, moderate $(n=6,18.1 \% ; 5 \leq A H I<10)$, and severe $(\mathrm{n}=12,36.5 \%$; AHI $\geq 10)$ OSAS.

The OSAS group included 26 males (78.7\%) and 7 females (21.3\%), which had more males compared with the control group [13 males (56.5\%), 10 females (43.5\%)]. There was no significant difference in age between the two groups $(\mathrm{p}=0.933)$. 
The tonsil size was significantly $(\mathrm{p}=0.002)$ greater in the OSAS group ( $6.52 \pm 0.90$ vs. $5.57 \pm 1.23)$. There was no significant difference in the AN ratio between the two groups $(\mathrm{p}=0.732)$. The $\mathrm{AHI}$ and oxygen desaturation index (ODI) in the OSAS group were significantly higher than in the controls, and there was no significant difference in the KMPESS between the two groups $(\mathrm{p}=0.646)$.

The levels of SR after PSG in both OSAS and control groups were higher than those before PSG, but there was no significant difference between the two groups for each level of SR before and after PSG, the subtraction of SRs, and ratio of SRs between before and after PSG (Table 1).

Moreover, there was no significant difference between the control group and the three OSAS severity subgroups for each level of SR before and after PSG, the subtraction of SRs, and ratio of SRs between before and after PSG. Additionally, in comparison with the control group and each OSAS severity group, the salivary parameters of each OSAS subgroup were not significantly different compared with those of the controls (Table 2). The tonsil size and the AN ratio in OSAS groups did not show any correlation with the SR parameters (Table 3), and the SR parameters were not related to the KMPESS, the lowest oxygen saturation, and ODI in the OSAS group (Table 4 and 5).

\section{DISCUSSION}

OSA is caused by upper airway collapse during sleep, which results in chronic intermittent hypoxia and sleep fragmentation [15]. Recent studies have reported that fragmented sleep might be correlated with an increase in oxidative stress mediators and that the intermittent hypoxia by repeated breathing cessation also causes oxidative stress with the production of reactive oxygen species (ROS) [5]. The increased levels of ROS

Table 1. Basic demographic, anthropometric, polysomnographic, questionnaire data of studied patients

\begin{tabular}{|c|c|c|c|c|}
\hline & Total $(\mathrm{n}=56)$ & Control $(\mathrm{n}=23)$ & OSAS $(n=33)$ & $\mathrm{p}$-value \\
\hline Gender & & & & 0.075 \\
\hline Male & $39(69.6)$ & $13(56.5)$ & $26(78.7)$ & \\
\hline Female & $17(30.4)$ & $10(43.5)$ & $7(21.3)$ & \\
\hline Age (yr) & $7.04(2.62)$ & $7.0(2.82)$ & $7.06(2.51)$ & 0.933 \\
\hline BMI $\left(\mathrm{kg} / \mathrm{m}^{2}\right)$ & $18.63(3.20)$ & $18.07(3.13)$ & $19.01(3.24)$ & 0.287 \\
\hline Tonsil size & $6.13(1.14)$ & $5.57(1.23)$ & $6.52(0.90)$ & $0.002^{*}$ \\
\hline AN ratio & $0.59(0.13)$ & $0.58(0.18)$ & $0.6(0.1)$ & 0.732 \\
\hline $\mathrm{AHI}$ & $7.06(11.21)$ & $0.19(0.2)$ & $11.85(12.59)$ & $<0.0001^{*}$ \\
\hline $0 \leq \mathrm{AHI}<1$ & $23(41)$ & $23(100.0)$ & $0(0)$ & $<0.0001^{*}$ \\
\hline $1 \leq \mathrm{AHI}<5$ & $15(26.7)$ & $0(0.0)$ & $15(45.4)$ & \\
\hline $5 \leq \mathrm{AHI}<10$ & $6(10.7)$ & $0(0.0)$ & $6(18.1)$ & \\
\hline $10 \leq \mathrm{AHI}$ & $12(21.6)$ & $0(0.0)$ & $12(36.5)$ & \\
\hline \multicolumn{5}{|l|}{ Resistin (ng/mL) } \\
\hline Night & $4.47(3.37)$ & $4.99(3.81)$ & $4.10(3.03)$ & 0.340 \\
\hline Morning & $8.12(7.33)$ & $7.52(3.58)$ & $8.55(9.11)$ & 0.609 \\
\hline Subtract & $3.66(7.56)$ & $2.53(4.64)$ & $4.44(9.04)$ & 0.356 \\
\hline Ratio & $2.70(2.44)$ & $2.55(2.53)$ & $2.81(2.48)$ & 0.700 \\
\hline REM_AHI & $15.38(27.40)$ & $0.89(0.93)$ & $25.48(32.15)$ & $0.001^{*}$ \\
\hline NREM_AHI & $5.25(8.96)$ & $0.03(0.06)$ & $8.89(10.23)$ & $<0.0001^{*}$ \\
\hline ODI & $4.80(17.27)$ & $0.21(0.30)$ & $8.0(22.07)$ & 0.097 \\
\hline Lowest_SaO ${ }_{2}(\%)$ & $88.54(13.41)$ & $87.19(19.88)$ & 89.48 (5.93) & 0.534 \\
\hline KMPESS & $5.09(3.47)$ & $5.35(3.47)$ & $4.91(3.51)$ & 0.646 \\
\hline
\end{tabular}

Categorical variables tested by chi-square test and Fisher's exact test are expressed as $\mathrm{n}(\%)$, and quantitative variables tested by $\mathrm{t}$-test are expressed as mean (standard deviation).

${ }^{*} \mathrm{p}<0.05$.

OSAS: obstructive sleep apnea syndrome, BMI: body mass index, AHI: apnea hypopnea index, AN ratio: ratio of the maximal adenoid thickness to the distance along a line from the posterior-superior edge of the hard palate to the spheno-occipital synchondrosis on the base of the skull, Resistin Night: measurements before PSG, Resistin Morning: measurements after PSG, Resistin Subtraction: subtraction of the measurements before PSG from the measurements after PSG, Resistin Ratio: ratio of the measurements after PSG to the measurements before PSG, REM_AHI: AHI during REM sleep, NREM_AHI: AHI during non-REM sleep, ODI: oxygen desaturation index, KMPESS: score in the Korean version of modified pediatric Epworth Sleepiness Scale. 
Table 2. The measurement regarding the salivary resistin according to the OSAS severity subgroup

\begin{tabular}{|c|c|c|c|c|c|c|c|c|}
\hline & \multicolumn{4}{|c|}{ OSAS severity subgroup } & \multirow[b]{2}{*}{ p-value* } & \multirow[b]{2}{*}{$\begin{array}{c}0 \text { vs. } 1 \\
\text { p-value }^{\dagger}\end{array}$} & \multirow[b]{2}{*}{$\begin{array}{c}0 \text { vs. } 2 \\
\text { p-value }\end{array}$} & \multirow[b]{2}{*}{$\begin{array}{c}0 \text { vs. } 3 \\
\text { p-value }\end{array}$} \\
\hline & $\begin{array}{c}\text { Normal }(0) \\
0 \leq \mathrm{AHI}<1 \\
(\mathrm{n}=23)\end{array}$ & $\begin{array}{c}\text { Mild (1) } \\
1 \leq \text { AHI < } 5 \\
(\mathrm{n}=15)\end{array}$ & $\begin{array}{c}\text { Moderate (2) } \\
5 \leq \mathrm{AHI}<10 \\
(\mathrm{n}=6)\end{array}$ & $\begin{array}{c}\text { Severe }(3) \\
10 \leq A H I \\
(n=12)\end{array}$ & & & & \\
\hline \multicolumn{9}{|c|}{ Resistin (ng/mL) } \\
\hline Night & $4.96(3.73)$ & $3.23(2.24)$ & $5.78(4.30)$ & $4.24(3.14)$ & 0.564 & 0.273 & 0.618 & 0.771 \\
\hline Morning & $7.42(3.53)$ & $7.20(5.18)$ & $15.68(18.80)$ & $6.80(3.85)$ & 0.840 & 0.425 & 0.979 & 0.572 \\
\hline Subtract & $2.46(4.56)$ & $3.97(5.00)$ & $9.90(19.20)$ & $2.56(4.40)$ & 0.990 & 0.930 & 0.937 & 0.797 \\
\hline Ratio & $2.48(2.49)$ & $3.23(2.74)$ & $2.93(3.49)$ & $2.39(1.70)$ & 0.940 & 0.701 & 0.979 & 0.595 \\
\hline
\end{tabular}

${ }^{*} \mathrm{p}$ value: $\mathrm{p}$ value of difference between normal vs. Mild vs. Moderate vs. Severe by Kruskal-Wallis test. ${ }^{\dagger} \mathrm{p}$ value: $\mathrm{p}$ value of difference between normal vs. Mild by Wilcoxon rank-sum test. ${ }^{\ddagger} \mathrm{p}$ value: $\mathrm{p}$ value of difference between normal vs. Moderate by Wilcoxon rank-sum test. ${ }^{\S} \mathrm{p}$ value: $\mathrm{p}$ value of difference between normal vs. Severe by Wilcoxon rank-sum test.

OSAS: obstructive sleep apnea syndrome, AHI: apnea hypopnea index.

Table 3. The relationship with the measurements for the salivary resistin and the degree of upper airway obstruction

\begin{tabular}{|c|c|c|c|c|c|c|}
\hline & \multicolumn{6}{|c|}{ OSAS $(\mathrm{AHI}>1)(\mathrm{n}=33)$} \\
\hline & \multicolumn{3}{|c|}{ Tonsil size } & \multicolumn{3}{|c|}{ AN_ratio } \\
\hline & $\mathrm{n}$ & $\mathrm{r}$ & $\mathrm{p}$-value & $\mathrm{n}$ & $\mathrm{r}$ & p-value \\
\hline \multicolumn{7}{|l|}{ Resistin } \\
\hline Night & 33 & -0.327 & 0.063 & 24 & -0.055 & 0.799 \\
\hline Morning & 33 & 0.081 & 0.654 & 24 & 0.154 & 0.473 \\
\hline Subtract & 33 & 0.261 & 0.143 & 24 & 0.124 & 0.564 \\
\hline Ratio & 33 & 0.273 & 0.125 & 24 & 0.049 & 0.819 \\
\hline
\end{tabular}

Spearman's correlation.

OSAS: obstructive sleep apnea syndrome, AHI: apnea hypopnea index.

Table 4. The relationship with the measurements for the salivary resistin and the questionnaire

\begin{tabular}{lccc}
\hline & \multicolumn{3}{c}{ KMPESS } \\
\cline { 2 - 4 } & $\mathrm{n}$ & $\mathrm{r}$ & $\mathrm{p}$-value \\
\hline Resistin & 32 & 0.053 & 0.772 \\
Night & 32 & 0.156 & 0.393 \\
Morning & 32 & 0.122 & 0.507 \\
Subtract & 32 & 0.074 & 0.689 \\
Ratio & & \\
\hline
\end{tabular}

Spearman's correlation.

KMPESS: Korean version of modified pediatric Epworth Sleepiness Scale.

by abnormal sleep-related oxidative stress might cause systemic inflammation, which might result in the production of adipokines. Therefore, OSA can be considered to be closely related to systemic inflammation [16].

Resistin, an adipocyte-derived cytokine (adipokine), may contribute to the development of obesity, insulin resistance, and metabolic syndrome. Recently, many studies have reported the association between adipokines and OSAS, as well as between resistin and systemic inflammation. Yamamoto et al. [17] dem-
Table 5. The relationship with the measurements for the resistin and the lowest oxygen desaturation

\begin{tabular}{|c|c|c|c|c|c|}
\hline & \multirow{2}{*}{$\mathrm{n}$} & \multicolumn{2}{|c|}{ ODI } & \multicolumn{2}{|c|}{ Lowest $\mathrm{O}_{2}$ saturation } \\
\hline & & $\mathrm{r}$ & p-value & $\mathrm{r}$ & $\mathrm{p}$-value \\
\hline \multicolumn{6}{|l|}{ Resistin } \\
\hline Night & 33 & -0.108 & 0.551 & -0.035 & 0.848 \\
\hline Morning & 33 & 0.099 & 0.584 & -0.084 & 0.641 \\
\hline Subtract & 33 & 0.154 & 0.393 & -0.093 & 0.608 \\
\hline Ratio & 33 & 0.154 & 0.393 & -0.028 & 0.877 \\
\hline
\end{tabular}

Spearman's correlation.

ODI: oxygen desaturation index.

onstrated that OSA patients had higher levels of serum resistin than controls and that resistin was increased depending on OSA severity. Zycari et al. [5] also reported a significantly higher level of resistin in patients with snoring compared to the control group. These results indicate that OSA is independently associated with resistin, possibly mediating systemic inflammatory processes [17]. Based on previous results, it could be suggested that the markers for systemic inflammation and oxidative stress, such as resistin, play a significant role in the identification of children with SDB.

Currently, saliva testing has gained more popularity in the clinical assessment of hormonal perturbations, detection of HIV antibodies, DNA analysis, alcohol screening, and drug testing because saliva sampling is a non-invasive and relatively stressfree diagnostic alternative to blood sampling.

SR is not affected by eating activity and correlated with serum resistin levels at any time point of the oral glucose tolerance test, which suggests the potential diagnostic value of saliva in health screening and risk stratification studies, particularly in the pediatric population [18].

Although most studies involving SR used individual samples obtained at one time point, the level of resistin mRNA showed a circadian rhythm during a 24-h study period [19]. Additionally, the changes during sleep may be partly related to circadian 
factors, as the central and peripheral circadian clocks have been linked to both whole-body and organ-specific energy metabolism [15]. Considering the aforementioned observations, to explore the effect of SDB on SR during night, time of salivary sampling is an important factor as SR levels changed significantly during the 24-h study period [15].

In this study, we measured the SR levels at night before sleep (baseline result before sleep) and early morning after waking (result affected by events during sleep) at 2 time points. Although the results could not sufficiently reflect the diurnal pattern of SR during the 24-h period, it could reflect the effect of SDB on SR secretion during sleep. Moreover, in this study, ratios of SR were used to reduce individual differences.

However, contrary to our expectation, no significant differences were observed in each level of SR before and after PSG, the subtraction of SR, and the ratio of SR before and after PSG between the SDB and control groups and among the control group and three OSAS severity subgroups. Our results were different from those of previous studies showing that resistin production could be enhanced by hypoxic stress during sleep, possibly mediating systemic inflammatory processes [5,17]. Notably, similar discrepancy was observed in previous studies to show a contradictory (significant or non-significant) association of serum and SR levels with either body mass index or insulin sensitivity. They also elaborated on the possible reasons for discrepancy, such as the sample size in each study, different hormonal determination methods, and lack of adjustment to the effect of additional variables, which could explain the discrepancy of our results with those of previous studies $[10,20]$.

However, several studies have reported results different from those in the aforementioned papers. Tauman et al. [21] reported no significant differences in resistin levels as a function of AHI, unlike other adipokine such as adiponectin and leptin. Ursavas et al. [6] also showed that no significant difference was noted in the levels of leptin, adiponectin, and resistin in the OSAS group compared with the control group and that no significant correlation was observed among leptin, adiponectin, resistin, and any polysomnographic parameters. These results show a contradictory association between resistin and OSAS in previous studies and could support the clinical significance of our results for SR in pediatric SDB.

To the best of our knowledge, this study is the first to evaluate the relationship between SDB and SR in pediatric subjects. However, the study has some limitations; all factors affecting pediatric sleep apnea were not completely controlled, although they had a prospective nature. There were normal and snoring subjects in the control group, which might be heterogeneous and subjects of 2 groups were not appropriately sex-matched. Some PSG parameters, such as periodic limb movement index, were not used in this study. Although the current study suggested that SR has no correlation with AHI, KMPESS, the lowest oxygen saturation, and ODI, considering the discrepancy from some previous studies, further studies must be conducted.

The saliva sampling was easy, noninvasive, and pain-free method and suitable for multiple sampling, especially in children. This study with the SR showed that SR had no significant relationship with OSA related parameters like AHI, KMPESS, the lowest oxygen saturation, and ODI. However, considering inconsistency between resistin and OSAS, further studies should be conducted.

\section{Conflicts of Interest}

The authors have no financial conflicts of interest.

\section{Authors' Contribution}

Conceptualization: Park CS, Park DS. Data curation: Hwang JH, Kim IH, Lee HS. Formal analysis: Park CS. Project administration: Park CS. Resources: Park CS. Software: Park CS. Supervision: Park CS. Validation: Park CS. Visualization: Hwang JH. Writing_original draft: Park CS. Writing_review \& editing: Park CS.

\section{REFERENCES}

1. Kaditis AG, Alonso Alvarez ML, Boudewyns A, Alexopoulos EI, Ersu R, Joosten $\mathrm{K}$, et al. Obstructive sleep disordered breathing in 2- to 18-yearold children: diagnosis and management. Eur Respir J 2016;47:69-94.

2. Goldstein NA, Stefanov DG, Graw-Panzer KD, Fahmy SA, Fishkin S, Jackson A, et al. Validation of a clinical assessment score for pediatric sleep-disordered breathing. Laryngoscope 2012;122:2096-104.

3. Marcus CL, Brooks LJ, Draper KA, Gozal D, Halbower AC, Jones J, et al. Diagnosis and management of childhood obstructive sleep apnea syndrome. Pediatrics 2012;130:e714-55.

4. Dehlink E, Tan HL. Update on paediatric obstructive sleep apnoea. J Thorac Dis 2016;8:224-35.

5. Zicari AM, Cutrera R, Occasi F, Carbone MP, Cesoni Marcelli A, De Castro G, et al. Serum resistin levels in children with primary snoring. Int J Immunopathol Pharmacol 2014;27:449-54.

6. Ursavas A, Ilcol YO, Nalci N, Karadag M, Ege E. Ghrelin, leptin, adiponectin, and resistin levels in sleep apnea syndrome: role of obesity. Ann Thorac Med 2010;5:161-5.

7. Sales LV, Bruin VM, D’Almeida V, Pompéia S, Bueno OF, Tufik S, et al. Cognition and biomarkers of oxidative stress in obstructive sleep apnea. Clinics (Sao Paulo) 2013;68:449-55.

8. Zhang J, Veasey S. Making sense of oxidative stress in obstructive sleep apnea: mediator or distracter? Front Neurol 2012;3:179.

9. Garvey JF, Taylor CT, McNicholas WT. Cardiovascular disease in obstructive sleep apnoea syndrome: the role of intermittent hypoxia and inflammation. Eur Respir J 2009;33:1195-205.

10. Yin J, Gao H, Yang J, Xu L, Li M. Measurement of salivary resistin level in patients with type 2 diabetes. Int J Endocrinol 2012;2012:359724.

11. Park CS, Guilleminault C, Park HJ, Cho JH, Lee HK, Son HL, et al. Correlation of salivary alpha amylase level and adenotonsillar hypertrophy with sleep disordered breathing in pediatric subjects. J Clin Sleep Med 2014;10:559-66.

12. Park CS, Guilleminault C, Hwang SH, Jeong JH, Park DS, Maeng JH. Correlation of salivary cortisol level with obstructive sleep apnea syndrome in pediatric subjects. Sleep Med 2013;14:978-84.

13. Hwang SH, Guilleminault C, Park CS, Kim TW, Hong SC. Usefulness of adenotonsillar size for prediction of severity of obstructive sleep apnea and flow limitation. Otolaryngol Head Neck Surg 2013;149:326-34.

14. Fujioka M, Young LW, Girdany BR. Radiographic evaluation of adenoidal size in children: adenoidal-nasopharyngeal ratio. AJR Am J Roentgenol 1979;133:401-4.

15. Sánchez-de-la-Torre M, Barceló A, Piérola J, de la Peña M, Valls J, Barbé F. Impact of obstructive sleep apnea on the 24-h metabolic hor- 
mone profile. Sleep Med 2014;15:625-30.

16. Inancli HM, Enoz M. Obstructive sleep apnea syndrome and upper airway inflammation. Recent Pat Inflamm Allergy Drug Discov 2010;4: 54-7.

17. Yamamoto Y, Fujiuchi S, Hiramatsu M, Nishigaki Y, Takeda A, Fujita Y, et al. Resistin is closely related to systemic inflammation in obstructive sleep apnea. Respiration 2008;76:377-85.

18. Desai GS, Mathews ST. Saliva as a non-invasive diagnostic tool for inflammation and insulin-resistance. World J Diabetes 2014;5:730-8.
19. Oliver P, Ribot J, Rodríguez AM, Sánchez J, Picó C, Palou A. Resistin as a putative modulator of insulin action in the daily feeding/fasting rhythm. Pflugers Arch 2006;452:260-7.

20. Mamali I, Roupas ND, Armeni AK, Theodoropoulou A, Markou KB, Georgopoulos NA. Measurement of salivary resistin, visfatin and adiponectin levels. Peptides 2012;33:120-4.

21. Tauman R, Serpero LD, Capdevila OS, O'Brien LM, Goldbart AD, Kheirandish-Gozal L, et al. Adipokines in children with sleep disordered breathing. Sleep 2007;30:443-9. 